\title{
CORRESPONDENCE
}

\section{Tanshinone II A: a Potent, Natural Anti-carcinogenic Agent for the Management of Systemic Malignancies}

\author{
Shailendra Kapoor
}

To the editor: I read with great interest the recent article by $\mathrm{Li}$, et $\mathrm{al}^{(1)}$. The authors have clearly shown that tanshinone II A inhibits myocardial cell hypertrophy induced by angiotensin II by inhibiting p-ERK1/2 expression. Interestingly, tanshinone II $A$ also has potent anti-carcinogenic properties. As a result, tanshinone $I I A$ is rapidly emerging as an agent with considerable promise in the field of oncology.

For instance, Wang, et $\mathrm{al}^{(2)}$ and Dong, et $\mathrm{al}^{(3)}$ have shown that tanshinone II $A$ alters the expression of ADPRTL1 and thereby attenuates tumor growth as well as metastasis in breast cancer cells as well as in neurological tumors such as gliomas. Tanshinone II A also decreases the expression of the bcl-2 gene and thereby causes $\mathrm{G}_{2} / \mathrm{M}$ arrest and subsequent inhibition of tumor growth in gastric carcinomas ${ }^{(4)}$. Tanshinone II $\mathrm{A}$ is also active in inducing apoptosis in leukemias such as acute promyelocytic leukemias ${ }^{(5)}$. Tanshinone II A also decreases the proliferation of vascular smooth muscle cells and may thus have an additional role in attenuating tumor growth in highly vascular malignancies ${ }^{(6)}$. Similarly, tanshinone II A down-regulates the expression of nucleophosmin in the nucleus of osteosarcoma cells and thereby has a negative effect on growth of these tumors ${ }^{(7)}$. Similarly, exposure of HepG2 cell lines to tanshinone II A for a period of $24 \mathrm{~h}$ decreases $\mathrm{G}_{1}$ cells by almost $23 \%$, clearly demonstrating the efficacy of tanshinone II A as a potent apoptotic agent in these cells ${ }^{(8,9)}$.

Besides these anti-carcinogenic properties, tanshinone II A also decreases the synthesis of interleukin-6 and thus plays a modulating role in systemic inflammatory processes ${ }^{(10)}$. For instance, it attenuates inflammatory hepatic damage secondary to concanavalin $A^{(11)}$. As is clear from the above examples, tanshinone II $\mathrm{A}$ is a potent anticarcinogenic agent which has a promising future in oncology. There is a clear and urgent need for further large scale studies to evaluate its efficacy in other malignancies as well as a need to increase awareness about its beneficial applications amongst oncologists.

\section{REFERENCES}

1. Li SS, Feng J, Zheng Z, Liang QS. Effect of sodium tanshinone II A sulfonate on phosphorylation of extracellular signalregulated kinase 1/2 in angiotensin II -induced hypertrophy of myocardial cells. Chin J Integr Med 2008;14:123-127.

2. Wang X, Wei Y, Yuan S, Liu G, Lu Y, Zhang J, et al. Potential anticancer activity of tanshinone II A against human breast cancer. Int J Cancer 2005;116:799-807.

3. Dong X, Dong J, Peng G. Growth-inhibiting and apoptosisinducing effects of tanshinone II A on human gastric carcinoma cells. J Huazhong Univ Sci Technol Med Sci (Chin) 2007;27:706-709.

4. Efferth T, Kahl S, Paulus K, Adams M, Rauh R, Boechzelt $\mathrm{H}$, et al. Phytochemistry and pharmacogenomics of natural products derived from traditional Chinese medicine and Chinese materia medica with activity against tumor cells. Mol Cancer Ther 2008;7:152-161.

5. Liu JJ, Lin DJ, Liu PQ, Huang M, Li XD, Huang RW. Induction of apoptosis and inhibition of cell adhesive and invasive effects by tanshinone II A in acute promyelocytic leukemia cells in vitro. J Biomed Sci 2006;13:813-823.

6. Zhang $\mathrm{HH}$, Chen YC, Liang L, Zeng Z. Tanshinone II $A$ inhibits in vitro cellular proliferation and migration of vascular smooth muscle cells of rabbits. J Sichuan Univ (Med Sci Edit, Chin) 2008;39:188-192.

7. Li QF, Shi SL, Liu QR, Tang J, Song J, Liang Y. Anticancer effects of ginsenoside Rg1, cinnamic acid, and tanshinone II A in osteosarcoma MG-63 cells: nuclear matrix downregulation and cytoplasmic trafficking of nucleophosmin. Int J Biochem Cell Biol 2008;40:1918-1929.

8. Lee WY, Chiu LC, Yeung JH. Cytotoxicity of major tanshinones isolated from Danshen (Salvia miltiorrhiza) on HepG2 cells in relation to glutathione perturbation. Food Chem Toxicol 2008;46:328-338.

9. Zhong ZH, Chen WG, Liu YH, Li QX, Qiu Y. Inhibition of cell growth and induction of apoptosis in human hepatoma cell line HepG2 by tanshione II A. J Central South Univ (Med Sci Edit, Chin) 2007;32:99-103.

10. Guo WQ, Xu WJ, Wang T, Chang JL, Chen MJ, He GY. Tanshinone II -A, a putative modulator can regulate pro-inflammatory and anti-inflammatory cytokines in inflammatory conditions in RAW264.7 cells. Biomedicine \& Pharmacotherapy Available online 11 July 2008.

11. Xu Y, Feng D, Wang Y, Lin S, Xu L. Sodium tanshinone II, a sulfonate protects mice from ConA-induced hepatitis via inhibiting NF-kappaB and IFN-gamma/STAT1 pathways. J Clin Immunol 2008; 28:512-519.

(Received August 27, 2008) Edited by TAO Bo

University of Illinois at Chicago, 75 Kristin Circle \#413 Schaumburg, IL 60195 Chicago, USA

Tel: 001-847-885-7896, E-mail: shailendrakapoor@yahoo.com DOI: 10.1007/s11655-009-0153-5 